\title{
Pour une histoire sociale de l'écriture
}

Histoire et génétique textuelle

\section{Philippe Artières}

\section{(2) OpenEdition}

Journals

Édition électronique

URL : https://journals.openedition.org/genesis/137

DOI : $10.4000 /$ genesis. 137

ISSN : 2268-1590

Éditeur :

Presses universitaires de Paris Sorbonne (PUPS), Société internationale de génétique artistique littéraire et scientifique (SIGALES)

\section{Édition imprimée}

Date de publication : 20 juin 2010

Pagination : 185-189

ISBN : 978-2-84050-697-3

ISSN : 1167-5101

\section{Référence électronique}

Philippe Artières, «Pour une histoire sociale de l'écriture », Genesis [En ligne], 30 | 2010, mis en ligne le 30 mai 2012, consulté le 30 mars 2023. URL : http://journals.openedition.org/genesis/137 ; DOI : https://doi.org/10.4000/genesis. 137 


\title{
Pour une histoire sociale de l'écriture. Histoire et génétique textuelle
}

\author{
Philippe Artières*
}

En souvenir de Gilles Cugnon

$\mathrm{D}$ ans un compte rendu pour les Annales en 1964, Michel Foucault soulignait que le XIXe siècle avait inventé la conservation documentaire absolue en créant, avec les archives et la bibliothèque, « un fonds de langage stagnant » fait de brouillons, de fragments, et de griffonnages. En poursuivant la perspective de l'auteur des Mots et les choses sur ce langage inédit - qui, selon lui, n'est pas une addition à l'opus ou à la biographie de l'auteur mais un troisième objet absolument irréductible ${ }^{1}$, on inscrira la génétique textuelle dans ce contexte moderne de production de ce nouvel objet. Science des manuscrits, elle participe en effet d'une histoire de la lecture des écrits qui apparaît et se développe à un moment donné de l'histoire de la critique littéraire. Elle peut ainsi être associée à certains savoirs philologiques, mais aussi à une histoire des techniques et des supports. Aussi la génétique textuelle est-elle pour l'historien d'abord un objet d'histoire. Il est en effet fort probable qu'à cette manière spécifique de lire les ratures, d'interroger les biffures, bref, à tout ce savoir succédera un jour une autre façon d'appréhender ces matériaux et ces questions de genèse. L'ITEM est pour l'historien avant tout un lieu d'observation et de collecte de matériaux pour une histoire des savoirs sur l'écriture.

On peut alors étudier le travail des chercheurs de l'ITEM de la même manière et suivant les mêmes procédures que celles convoquées pour caractériser le regard des médecins sur les manuscrits de leurs patients à partir des années 1850 . Il ne s'agit pas de confondre ces deux regards mais de signaler combien ils présentent parfois des problématiques communes qui dessinent ensemble notre regard moderne sur l'écrit. L'extrême et inédite attention qu'y portaient psychiatres et médecins légistes révèle une généalogie méconnue de notre analyse des brouillons et des archives ${ }^{2}$; ce regard découvrait le manuscrit comme objet susceptible d'investigation, comme lieu de recherche.

Mais on doit dans ce même mouvement se demander quelle contribution la génétique textuelle est en mesure de livrer à une histoire sociale. Plus précisément, quels apports peut-on tirer de la génétique textuelle lorsqu'on entreprend une histoire sociale de l'écriture ?

\section{Obstacles}

Pour le chercheur en sciences sociales, le recours à la génétique textuelle pose en premier lieu deux problèmes épistémologiques connus, et ce, à travers les notions d'auteur et de genèse. Parce que l'historien des pratiques d'écriture travaille autant que faire se peut sur une série d'écrits émanant de plusieurs scripteurs, il est placé dans une posture délicate lorsqu'il se retrouve à vouloir appliquer à l'auteur d'un texte unique, souvent autobiographique, ce que les généticiens ont construit pour des écrivains. Faut-il pour autant renoncer à user de certains de leurs outils lorsque la notion d'auteur n'est pas opérante ? Deux voies qui se rejoignent s'ouvrent alors : adopter le même soin à singulariser les écrits d'un

\footnotetext{
* Cet article doit beaucoup aux échanges que nous avons au sein du groupe Anthropologie de l'écriture (IIAC, EHESS) animé par Béatrice Fraenkel. Mes remerciements à Annick Arnaud pour son attentive relecture. 1. Voir Michel Foucault, Dits et écrits, Édition établie par Daniel Defert et François Ewald, Paris, Gallimard, 1995, t. I, p. 457.

2. Voir notre article «Manuscrits ordinaires et savoir médical au XIX siècle », Genesis, n 15, 2000, p. 109-120.
} 
inconnu que ceux d'un Flaubert ; c'est-à-dire mettre en avant une série de traits scripturaux caractéristiques de ce scripteur par rapport aux pratiques de ses contemporains. Cela suppose, en somme, et la chose est moins aisée qu'il n'y paraît, d'octroyer à un inconnu la mention d'auteur. C'est exactement cette approche que nous avions adoptée pour étudier la genèse de l'autobiographie d'un apache de la Belle Époque, prénommé Émile Nouguier ${ }^{3}$. À partir $\mathrm{du}$ journal du jeune homme, mais surtout d'une première version de son récit de vie, l'étude montrait comment son autobiographie, intitulée «Les souvenirs d'un moineau », était le fruit d'un dispositif singulier de contrainte.

L'autre possibilité est à l'inverse de considérer que ce scripteur ordinaire a produit avec ses contemporains un corpus collectif qu'il convient d'analyser comme un ensemble, exactement comme pour les écrits d'un même scripteur. Autrement dit, l'opération consiste à désingulariser à l'extrême et à estimer que tous les scripteurs ont des pratiques quasi semblables selon la période à laquelle ils ont vécu. Dans cette perspective, suivie par exemple par Béatrice Fraenkel dans son travail sur la signature, les pratiques d'écriture sont analysées comme des actes d'écriture ${ }^{4}$. Fraenkel ne neutralise pas l'auteur, mais elle fait jouer celui-ci avec d'autres paramètres. On voit ainsi très bien combien cette approche de l'acte de signer dépasse largement une histoire de la littérature pour esquisser une histoire du sujet en Occident.

Le deuxième obstacle est moins visible mais beaucoup plus difficile à franchir car il relève de ce qui est à l'œuvre et au centre de la critique littéraire : la notion de création. Pour le chercheur en sciences sociales, la distinction entre le discours littéraire et les autres discours est le résultat d'un partage : suivant les périodes historiques et les ères géographiques, tel ou tel texte est considéré comme littéraire. C'est une qualification, une distinction historiquement déterminée. Pour l'historien, peu importe que les matériaux sur lesquels il travaille soient considérés comme littéraires ; ils sont d'abord des productions discursives, produit de pratiques sociales communes. L'écart n'est donc à ses yeux qu'un travail de distinction par rapport à une norme. Aussi, et cela va de pair, la notion de genèse est-elle pour lui particulièrement étrangère, voire problématique. La question est en effet, pour l'historien après Foucault, moins celle de l'origine d'un écrit que des conditions d'émergence de pratiques discursives. Il n'y a pas de ce point de vue mystère autour de la genèse d'un écrit, pas de moment magique qui échapperait et que le chercheur par une analyse très précise mettrait en lumière. La rature n'est donc pas pour lui un objet d'analyse, un écrit appartient à une série que le chercheur doit mettre en lumière. En d'autres termes, les pratiques d'écriture d'une Albertine Sarrazin (usage de supports minuscules, recours au fragment, etc.) sont, à ses yeux, à mettre davantage en relation avec les pratiques d'écriture en détention qu'avec celle d'une Violette Leduc. De même, la genèse des œuvres d'un Michel Leiris est peutêtre plus, aux yeux de l'historien, à rapprocher de celle des travaux ethnographiques (ceux de Leiris mais aussi de ses collègues contemporains, Roger Bastide par exemple5), en somme d'une écriture professionnelle. Cela ne signifie pas que la question de la genèse est non opérante, mais qu'elle est posée immédiatement en des termes collectifs et neutres : qu'est-ce qu'écrire ? Quels sont les actes qui composent ce que l'on désigne par le terme d'écriture ? Comment s'articulent-ils les uns aux autres ? Comment commence-ton ? Les archives des écrivains apparaissent ainsi comme une formidable banque d'échantillons graphiques pour l'historien des écritures.

Cette double banalisation de l'auteur et de la genèse - respectivement en scripteur et en actes d'écriture, pour reprendre les termes de Béatrice Fraenkel - n'indique pas pour autant que tout usage de la génétique textuelle hors du champ littéraire, notamment pour une histoire de l'écriture, est malvenu. Au contraire, devrions-nous dire : une fois opérée cette neutralisation et du sacre de l'écrivain et de la fétichisation de ses écritures, la génétique textuelle offre, d'une part des outils particulièrement utiles à l'historien, d'autre part ouvre une série de perspectives très stimulantes.

3. Voir «Écriture contrainte. Émile Nouguier, "Les souvenirs d'un moineau" ", dans P. Lejeune et C. Viollet (dir.), Genèses $d u$ « je », Paris, CNRS Éditions, 2000, p. 34-59.

4. Voir les travaux de Béatrice Fraenkel, qui renouvellent considérablement l'anthropologie de l'écriture, et sur ce point précis : "Signature et force graphique. Pour une pragmatique de l'écrit », dans La Lettre de l'AREHESS, $\mathrm{n}^{\circ}$ 31, septembre-décembre 2003, p. 14-24.

5. Sur ce point, voir le travail de Mathias Dreyfuss, « De l'écriture à l'archive », Master 2 sous la direction de B. Fraenkel, EHESS, septembre 2006. 


\section{Outils}

La génétique textuelle mobilise en effet plusieurs outils, précieux pour la perspective d'une histoire sociale de l'écriture, de description de l'objet écrit, avec en premier lieu une expertise codicologique, particulièrement performante, qu'elle emprunte aux paléographes et à la philologie. L'intérêt pour les papiers, leur histoire, mais également pour les encres, qui a pour visée en génétique de déterminer l'antériorité de telle pièce par rapport à telle autre, mais aussi l'authenticité d'un document, est une formidable contribution à l'histoire des objets écrits. Ce souci d'identification et de désignation des supports, qui fait la part belle à la matérialité de l'écrit, a pour conséquence de déplacer le regard vers le corps du scripteur et de s'interroger sur sa gestuelle. Déterminer et identifier très précisément le lieu de l'écriture, pouvoir repérer les ustensiles, telles sont en particulier les opérations très précieuses pour ceux qui travaillent par exemple sur les pratiques d'écriture anonymes. Sans tout ce savoir, l'appréhension d'un objet comme la lettre anonyme est en effet impossible. Il ne s'agit pas pour autant de se métamorphoser en policier traquant les scripteurs illicites, mais de comprendre comment par exemple cette pratique sociale du ressentiment ${ }^{6}$ a été rendue possible et surtout comment procèdent ces individus pour devenir anonymes. Quelles encres, quels papiers utilisent-ils ? On découvre ainsi que le support utilisé ne l'est jamais par hasard : de même que l'auteur de graffiti choisit son mur, l'auteur d'une missive anonyme décide du papier, de la couleur de l'encre, etc. Ce qui menace n'est pas seulement le message (« ta maison sautera le 1er mai ») mais la graphie et plus encore la feuille de papier... Ce n'est plus ici la codicologie qui doit être mobilisée, mais une sémiotique des manuscrits que la critique génétique a beaucoup nourrie depuis vingt ans ${ }^{7}$. L'espace de la page a ainsi été cartographié par les généticiens, qu'il s'agisse de la marge, des bas de page, ou encore du verso.

Il faut immédiatement ajouter que ce savoir génétique s'accompagne d'un lexique permettant aussi une description de l'objet écrit. C'est sans nul doute à partir de l'analyse et surtout de la description de cas littéraires que s'est imposé et généralisé progressivement un vocabulaire de l'écrit jusque-là confidentiel ${ }^{8}$ : décrire l'écrit sous toutes ses formes chez les écrivains - avec ses paperoles, ses biffures, etc. - pour être en mesure de décrire avec le même soin les manuscrits des hommes ordinaires. La diffusion de ce vocabulaire est surtout essentielle dans une visée comparative. Désormais, on peut, à partir de notices descriptives des manuscrits, comparer des pratiques.

Enfin, les techniques de transcription des manuscrits mises au point par les généticiens sont parmi les principaux outils utiles à l'historien. Longtemps, les écrits de l'homme ordinaire ont été réduits à la fonction documentaire, faute de moyens de transcription adéquats. Les écrits de l'homme du commun étaient corrigés, rectifiés. Songeons ici aux erreurs de transcription faites sur le manuscrit de Pierre Rivière au moment de sa publication dans la collection «Archives » de Julliard/Gallimard en 1973, erreurs indiquées par Philippe Lejeune dans un article de la revue Le Débat ${ }^{9}$. Les protocoles de transcription mis en place par les chercheurs en génétique textuelle permettent en effet, lorsque l'historien est confronté aux archives, comme ce fut mon cas avec des autobiographies de criminels, de déplacer le regard en s'intéressant à l'écrit-trace. La transcription dite diplomatique offre un outil pour « entendre » les textes avant de s'engouffrer dans un tunnel interprétatif. Travaillant sur le mémoire d'un inverti parricide de la fin du siècle, Charles Double, la transcription selon les règles, avec maintien de l'orthographe, de la syntaxe et de la mise en page du cahier original offrait une lecture du texte insoupçonnable. Ce récit, qui apparaissait au premier abord comme très scolaire, s'avérait à la lecture des ratures, des ajouts et de l'usage des marges un véritable exercice de résistance au pouvoir médical ${ }^{10}$. Mais la critique génétique apporte aussi une dimension que la transcription diplomatique des élèves de l'École des Chartes n'offre pas : l'acte d'écriture en tant qu'il relève d'un geste physique,

6. Voir notre article : « Des mots pour faire peur. Des lettres de menace à Paris en 1892 », Terrain, n 43, septembre 2004, p. 31-46.

7. Voir Genesis, n 10, « Sémiotique », 1996.

8. Voir les travaux de Claire Bustarret (IIAC, CNRS-EHESS). Voir aussi Michel Contat et Daniel Ferrer (dir.), Pourquoi la critique génétique? Méthodes, théories, Paris, CNRS Éditions, 1998.

9. «Lire Pierre Rivière », Le Débat, n 66, septembre-octobre 1991, p. 92 .

10. Voir « En quête d'un regard », postface de notre Livre des vies coupables, Paris, Albin Michel, 2000. 
d'une pratique corporelle. Pour la génétique textuelle, il y a des écritures tremblées, des taches, des trébuchements de la plume.

\section{Perspectives}

Enfin, parmi les analyses produites par les généticiens, il en est certaines qui, croyons-nous, sont particulièrement fécondes pour une histoire sociale de l'écriture. Pour la fin du XIXe siècle, le cas Zola est à bien des égards passionnant. Songeons ici par exemple à ses carnets d'enquêtes et de reportages étudiés par Henri Mitterand. À travers ces pages, s'esquisse un chapitre de l'histoire de la prise de notes, de cette curieuse pratique qui consiste non à dessiner ce qu'on voit mais à l'écrire. Ces analyses sont en effet à mettre en relation avec toutes les techniques graphiques d'enregistrement contemporaines, comme la sténographie étudiée par Delphine Gardey ${ }^{11}$.

Mais la lecture génétique invite également à lire tout autrement qu'on ne l'a fait jusqu'alors les écritures de gouvernement, qui sont un chapitre mal connu de l'histoire de l'écriture. L'historien Yves Cohen a fait une analyse des lettres de Staline dans cette perspective d'une gouvernementalité par l'écrit, en montrant que le «petit père des peuples » faisait véritablement présence par ces écrits, s'y donnant à voir physiquement ${ }^{12}$. Cet historien du travail, qui développe des analyses en pragmatique, est très attentif aux moindres accidents de la plume. On pourrait, me semble-t-il, travailler dans le même sens sur toute une série d'écrits de gouvernement, qu'ils soient locaux ou nationaux, infimes ou colossaux.

En faisant du manuscrit non plus seulement la trace d'une série d'actes mais l'acte même, la génétique compose des dossiers qui sont autant couches d'écritures que strates d'événements, des palimpsestes de pratiques. Ces «dossiers génétiques » que le chercheur constitue sont extrêmement intéressants à exporter hors du champ littéraire. On peut en effet interroger des pratiques scientifiques, notamment d'expertises, en composant de tels dossiers pour quelques affaires à partir des archives conservées ${ }^{13}$. On voit ainsi comment l'écriture n'est pas seulement le support d'une pratique de gouvernement, mais qu'elle est l'exercice même du pouvoir. L'analyse du dossier génétique d'une expertise criminelle le fait apparaître comme étant une succession d'actes, parfois contradictoires, où qui plus est plusieurs plumes interviennent.

C'est cette double dimension polygraphique et cumulative que l'on retrouve lorsqu'on importe le principe du dossier génétique pour analyser un dispositif d'apprentissage du pouvoir, comme celui à l'œuvre dans le premier journal du prince de Joinville datant des années 1830. Le fils de Louis-Philippe est embarqué sur un bateau de la marine royale pour apprendre la navigation, mais aussi l'exercice du pouvoir. Cette expérience de navigation en Méditerranée est l'objet d'une triple écriture : le journal quotidien que tient le jeune dauphin de 13 ans, les lettres qu'il envoie chaque jour à ses parents, enfin le rapport journalier de son tuteur. Le plus passionnant est que soixante-dix ans plus tard le prince de Joinville rédige à partir de ce dossier des souvenirs mais surtout produit une série de tableaux autobiographiques, faisant de ces événements des tableaux historiques, au même titre que la guerre civile américaine à laquelle il assista $^{14}$.

Cette étude du cas du prince de Joinville est exemplaire de cet usage possible de la génétique textuelle en histoire car, on l'a compris, le fils de Louis-Philippe nous intéresse moins comme auteur que comme un acteur ayant produit une série d'actes d'écriture sur des pratiques de navigation, de voyage et de gouvernement. L'analyse de ses manuscrits est à la fois une contribution à une histoire politique et à une histoire sociale de l'écriture.

11. Voir par exemple : Delphine Gardey, « Mécaniser l'écriture et photographier la parole : utopies, monde du bureau et histoires de genre et de techniques », Annales, Histoire, Sciences sociales, mai-juin 1999, n 3, p. 587-614.

12. Yves Cohen, «Des lettres comme action. Staline au début des années 1930 vu depuis le fonds Kaganovich », Cahiers du monde russe, $\mathrm{n}^{\circ}$ 38/3, 1997.

13. Voir notre article : «Les palimpsestes de l'expert », dans Sciences de la société, $\mathrm{n}^{\circ}$ 67, 2006, p. 33-43.

14. Philippe Artières et Bérénice Waty, « La dernière traversée du prince de Joinville. Du journal contraint à l'autobiographie peinte », dans Les Métamorphoses du journal personnel. De Rétif de la Bretonne à Sophie Calle, Catherine Viollet, Marie-Françoise Lemonnier-Delpy (dir.), Louvain-la-Neuve, Academia Bruylant, 2006, p. 29-44. 
Philippe Artières est historien, chercheur au CNRS-EHESS (équipe Anthropologie de l'écriture, IIAC, Paris).

Philippe Artières,ph.artieres@wanadoo.fr

\section{Pour une histoire sociale de l'écriture. Histoire et génétique textuelle}

La génétique textuelle témoigne, aux yeux de l'historien, un moment de l'histoire des savoirs sur l'écriture ; elle constitue également un outil pour interroger les archives, notamment privées, une fois levé un certain nombre d'obstacles épistémologiques. Ce savoir peut ainsi participer du programme d'une histoire sociale de l'écriture produite à partir des strates graphiques d'une époque.

For a historian, textual genetics testifies to a date in the history of knowledge on writing. It is also a tool for questioning archives, in particular private ones, after having removed a certain number of epistemological obstacles. This knowledge can thereby participate in the program of a social history of writing produced from the graphic layers of a given period.

Dem Historiker erscheint die Textgenetik als eine Etappe in der Geschichte der Schriftkunde. Sind einmal eine gewisse Anzahl von epistemologischen Hindernissen überwunden, so verfügt man zudem über ein Instrumentarium zur Archivforschung, insbesondere für Privatarchive. Sie kann so einen Beitrag zu einer Sozialgeschichte des Schreibens liefern, die sich mit der Erforschung der graphischen Schichten der Schriftlichkeit für eine bestimmte Epoche befasst.
La genética textual revela, en la perspectiva del historiador, un momento de la historia de los conocimientos sobre la escritura, al mismo tiempo que constituye una herramienta para interrogar los archivos, en particular los privados, una vez sorteada una cierta cantidad de obstáculos epistemológicos. Este saber puede participar así en un programa de una historia social de la escritura producida a partir de los estratos gráficos de una época.

A genética textual testemunha, aos olhos do historiador, um momento da história dos saberes da escrita; constitui igualmente um instrumento para interrogar os arquivos, nomeadamente privados, uma vez levantado certo número de obstáculos epistemológicos. Este saber pode assim participar do programa de uma história social da escrita, produzida a partir dos estratos gráficos de uma época.

La genetica testuale rappresenta, per gli storici, un momento della evoluzione del sapere intorno alla scrittura; oltre a costituire, una volta eliminati gli ostacoli di tipo epistemologico, uno strumento metodologico per lo studio archivistico, ed in particolare per lo studio degli archivi privati. Tale sapere può quindi inserirsi all'interno di un progetto di storia sociale della scrittura prodotta a partire dagli strati grafici di un'epoca. 